\title{
Nomenclatural corrections in the Rytidosperma complex (Danthonieae, Poaceae)
}

\author{
H. Peter Linder
}

\begin{abstract}
Linder, H. Peter (Bolus Herbarium, University of Cape Town, Rondebosch 7700, South Africa) 1997. Nomenclatural corrections in the Rytidosperma complex (Danthonieae, Poaceae). Telopea 7(3): 269-274. In a recent paper, Linder and Verboom proposed a new genus, Thonandia, but included the type of Notodanthonia Zotov in this new genus. Consequently, the species transferred to Thonandia should be under Notodanthonia, while the species placed by Linder and Verboom under Notodanthonia need a new generic name. The name Austrodanthonia Linder is proposed, and the relevant new combinations are made.
\end{abstract}

\section{Introduction}

Linder and Verboom (1996) proposed the dismemberment of the Australasian danthonioids into smaller genera, and proposed two new genera, Joycea and Thonandia, in addition to the existing genera, Notodanthonia and Rytidosperma. Other existing genera, like Pyrranthera, Erythranthera and Monostachya, were included in Rytidosperma. However, in error the type species of Notodanthonia, N. unarede, was included among the species of the new genus Thonandia, thus rendering this latter name invalid. This necessitates a new name for the genus Linder and Verboom called Notodanthonia, and all new combinations associated with that name. Fortunately, all the Thonandia species already have names in Notodanthonia, so no new combinations are needed in this genus.

\section{Nomenclature}

Austrodanthonia H.P. Linder, gen. nov., differt a ceteris generibus Danthoniearum callo saltem duplo longiore quam rachilla, a Danthonia D.C. et Joycea Linder hilo punctiformi, a Danthonia, Joycea et Notodanthonia Zotov indumento lemmatum plerumque fasciculato.

Type: Austrodanthonia caespitosa (Gaudich.) H.P. Linder

Caespitose or stoloniferous grasses with intra- or extravaginal innovations and truncate, two-awned prophylls, plants usually villous, occasionally glabrous. Ligules a single line of cilia, without contraligules or pseudopetioles. Inflorescence usually an open panicle, rarely contracted into a compact head. Spikelets 6-22 $\mathrm{mm}$ long, with 3-10 bisexual florets, disarticulating above the glumes. Callus villous, massive, at least twice as long as the rhachilla internode. Glumes always longer than the basal lemma, sometimes longer than the florets, with 3-13 veins, variously scaberulous or sparsely villous. Lemmas bilobed and 9-veined; indumentum generally in two rows of intercostal tufts on the back, occasionally glabrous or with the tufts reduced, or with a more or less continuous indumentum in the lower half, or with scattered indumentum between the rows of tufts; lateral lobes usually with setae; central awn usually with a 
basal, flattened, twisted column, and a hair-like apical portion, rarely reduced to a simpler structure. Palea 2-keeled, bilobed to truncate, often with hair-tufts on the flaps. Lodicules vascularised, usually bristly; anthers two; ovary with two widely separated styles. Caryopsis obovate, embryo-mark half the caryopsis length, hilum more or less punctate, $17-37 \%$ of the length of the caryopsis. Chromosome numbers 24-120.

This genus differs from Rytidosperma and Notodanthonia by the massive callus, which is always at least twice as longer as the rhachilla internode. It contains 28 species, from Australia, New Zealand and Malesia.

Austrodanthonia acerosa (Vickery) H.P. Linder, comb. nov.

Basionym: Danthonia acerosa Vickery, Contrib. New South Wales Natl Herb. 1: 296 (1950). Synonyms: Rytidosperma acerosum (Vickery) Connor \& Edgar, New Zealand J. Bot. 17: 331 (1979); Notodanthonia acerosa (Vickery) Veldkamp, Taxon 29: 296 (1980).

Type: Western Australia: Wooroloo, Dec 1907, Koch 1826; holo: NSW 2596; iso: K.

Austrodanthonia alpicola (Vickery) H.P. Linder, comb. nov.

Basionym: Danthonia semiannularis var. alpina Benth., Fl. Austral. 7: 595 (1878), non Buchanan (1872).

Synonyms: Danthonia alpicola Vickery, Contrib. New South Wales Natl Herb. 1: 297 (1950); Rytidosperma alpicolum (Vickery) Connor \& Edgar, New Zealand J. Bot. 17: 331 (1979); Notodanthonia alpicola (Vickery) Veldkamp, Taxon 29: 296 (1980).

Type: Victoria: Mt Buller, Mueller; lecto: K, fide Vickery, Contrib. NSW Natl Herb. 2: 300 (1956); iso: MEL.

Austrodanthonia auriculata (J.M. Black) H.P. Linder, comb. nov.

Basionym: Danthonia auriculata J.M. Black, Flora of South Australia, pt. 4: 674 (1929). Synonyms: Notodanthonia auricalata (J.M. Black) Zotov, New Zealand J. Bot. 1: 113 (1963); Rytidosperma auriculatum (J.M. Black) Connor \& Edgar, New Zealand J. Bot. 17: 322 (1979). Type: South Australia: Bundaleer hills, 11 Nov 1927, Black 2; lecto: AD, fide Vickery, Contrib. New South Wales Natl Herb. 2: 309 (1956); iso: K, MEL.

Austrodanthonia biannularis (Zotov) H.P. Linder, comb. nov.

Basionym: Notodanthonia biannularis Zotov, New Zealand J. Bot. 1: 116 (1963).

Synonym: Rytidosperma biannulare (Zotov) Connor \& Edgar, New Zealand J. Bot. 17: 324 (1979).

Type: New Zealand: Waitangi Forest, Northland, Zotov CHR 85021; holo: CHR.

Austrodanthonia bipartita (Link) H.P. Linder, comb. nov.

Basionym: Avena bipartita Link, Hort. Berol. 1: 113 (1827); Notodanthonia bipartita (Link) Veldkamp, Taxon 29: 296 (1980).

Synonyms: Danthonia linkii Kunth, Enum. Pl. 1: 315 (1833); Rytidosperma linkii (Kunth) Connor \& Edgar, New Zealand J. Bot. 17: 332 (1979); Notodanthonia linkii (Kunth) H.P. Linder, Telopea 6: 616 (1996), nom. illeg.

Type: B (non vide).

Austrodanthonia bonthainica (Jansen) H.P. Linder, comb. nov.

Basionym: Danthonia pilosa R. Br. var. bonthainica Jansen, Reinwardtia 2: 258 (1953).

Synonyms: Notodanthonia penicillata (R. Br.) Zotov ssp. bonthainica (Jansen) Veldkamp, Taxon 29: 298 (1980); Danthonia bonthainica (Jansen) Veldkamp, Blumea 38: 217 (1993); Notodanthonia bonthainica (Jansen) H.P. Linder, Telopea 6: 615 (1996).

Type: Celebes: Peak on Bonthaim, 2400-3000 m, Bünnemeijer 11971; holo BO; iso L. 
Austrodanthonia caespitosa (Gaudich.) H.P. Linder, comb. nov.

Basionym: Danthonia caespitosa Gaudich. in Freycinet, Voyage autour du Monde par des Corvettes l’Uranie et la Physicienne pendant 1817-1820, 4, Botanique: 408 (1829). Synonyms: Notodanthonia caespitosa (Gaudich.) Zotov, New Zealand J. Bot. 1: 117 (1963); Rytidosperma caespitosum (Gaudich.) Connor \& Edgar, New Zealand J. Bot. 17: 325 (1979). Type: Western Australia: Shark's Bay, Gaudichaud; holo: P; iso: K, BM.

Austrodanthonia carphoides (Benth.) H.P. Linder, comb. nov.

Basionym: Danthonia carphoides F. Muell. ex Benth., Fl. Austral. 7: 592 (1878).

Synonyms: Notodanthonia carphoides (Benth.) Zotov, New Zealand J. Bot. 1: 113 (1963); Rytidosperma carphoides (Benth.) Connor \& Edgar, New Zealand J. Bot. 17: 331 (1979).

Type: Victoria: Melbourne, 2 Nov 1853, Adamson 195; lecto: K, fide Vickery, Contrib. New South Wales Natl Herb. 2: 277 (1956). Residual syntypes: MacQuarie River, C. Moore; syn: K. New South Wales: Armidale, Perrott; syn: not found, ?K. New South Wales: Cavan nr Yass, Mrs Calvert; syn: K.

Austrodanthonia clavata (Zotov) H.P. Linder, comb. nov.

Basionym: Notodanthonia clavata Zotov, New Zealand J. Bot. 1: 119 (1963).

Synonym: Rytidosperma clavatum (Zotov) Connor \& Edgar, New Zealand J. Bot. 17: 326 (1979).

Type: New Zealand: Upper Hutt, Zotov CHR 79778; holo: CHR.

Austrodanthonia diemenica (D.I. Morris) H.P. Linder, comb. nov.

Basionym: Danthonia diemenica D.I. Morris, Muelleria 7: 153 (1990).

Synonym: Notodanthonia diemenica (D.I. Morris) H.P. Linder, Telopea 6: 616 (1996).

Type: Tasmania: Ouse River, Wild Dog Plains, 7 Jan 1983, Moscal 1292; holo: HO 65782.

Austrodanthonia duttoniana (Cashmore) H.P. Linder, comb. nov.

Basionym: Danthonia duttoniana Cashmore, Comm. Aust., C.S.I.R., Bull. 69 Appendix 2: 72 (1932).

Synonyms: Rytidosperma duttonianum (Cashmore) Connor \& Edgar, New Zealand J. Bot. 17: 332 (1979); Notodanthonia duttoniana (Cashmore) Veldkamp, Taxon 29: 296 (1980). Type: South Australia: Werribee, 2 Dec 1932, Cashmore Ga65; lecto: ADW (now at AD) fide Vickery, Contrib. New South Wales Natl Herb. 2: 304 ; iso: K, NSW 19682.

Austrodanthonia eriantha (Lindl.) H.P. Linder, comb. nov.

Basionym: Danthonia eriantha Lindl., Mitchell, Three Exped. Eastern Australia 2: 304 (1838). Synonyms: Rytidosperma erianthum (Lindl.) Connor \& Edgar, New Zealand J. Bot. 17: 323 (1979); Notodanthonia eriantha (Lindl.) Veldkamp, Taxon 29: 296 (1980).

Type: Australia: Interior of New Holland, Major Mitchell's Expedition 1836, ex herb. Lindley; holo: CGE, fragm. NSW 6960.

Austrodanthonia fulva (Vickery) H.P. Linder, comb. nov.

Basionym: Danthonia linkii Kunth var. fulva Vickery, Contrib. New South Wales Natl Herb. 1: 299 (1950).

Synonyms: Rytidosperma linkii var. fulvum (Vickery) Connor \& Edgar, New Zealand J. Bot. 17: 332 (1979); Notodanthonia bipartita (Link) Veldkamp var. fulva (Vickery) Veldkamp, Taxon 29: 296 (1980); Notodanthonia fulva (Vickery) H.P. Linder, Telopea 6: 616 (1996).

Type: New South Wales: Flemington, 31 Mar 1929, Vickery s.n.; holo: NSW 1573; iso: K.

Austrodanthonia geniculata (J.M. Black) H.P. Linder, comb. nov.

Basionym: Danthonia geniculata J.M. Black, Trans. \& Proc. Roy. Soc. South Australia 53: 261 (1929). 
Synonyms: Notodanthonia geniculata (J.M. Black) Zotov, New Zealand J. Bot. 1: 114 (1963); Rytidosperma geniculatum (J.M. Black) Connor \& Edgar, New Zealand J. Bot. 17: 323 (1979). Type: South Australia: Keith, 23 Nov 1917, Black 1; lecto: AD, fide Vickery, Contrib. New South Wales Natl Herb. 2: 279 (1956); iso: MEL, K, NSW 1377 \& 1378.

Austrodanthonia induta (Vickery) H.P. Linder, comb. nov.

Basionym: Danthonia induta Vickery, Contrib. New South Wales Natl Herb. 1: 298 (1950). Synonyms: Rytidosperma indutum (Vickery) Connor \& Edgar, New Zealand J. Bot. 17: 332 (1979); Notodanthonia induta (Vickery) Veldkamp, Taxon 29: 296 (1980).

Type: New South Wales: Armidale, Dec 1938, Ingram s.n.; holo: NSW 1344; iso: K.

Austrodanthonia laevis (Vickery) H.P. Linder, comb. nov.

Basionym: Danthonia laevis Vickery, Contrib. New South Wales Natl Herb. 1: 299 (1950). Synonyms: Notodanthonia laevis (Vickery) Zotov, New Zealand J. Bot. 1: 117 (1963); Rytidosperma laeve (Vickery) Connor \& Edgar, New Zealand J. Bot. 17: 325 (1979).

Type: Australian Capital Territory: base of Mt Stromlo, 26 April 1941, Melvaine s.n.; holo: NSW 3232.

Austrodanthonia mera (Connor \& Edgar) H.P. Linder, comb. nov.

Basionym: Rytidosperma merum Connor \& Edgar, New Zealand J. Bot. 17: 328 (1979). Synonym: Notodanthonia mera (Connor \& Edgar) H.P. Linder, Telopea 6: 616 (1996).

Type: New Zealand: Marlborough, Williams CHR 309413; holo: CHR.

Austrodanthonia monticola (Vickery) H.P. Linder, comb. nov.

Basionym: Danthonia monticola Vickery, Contrib. New South Wales Natl Herb. 1: 299 (1950). Synonyms: Rytidosperma monticolum (Vickery) Connor \& Edgar, New Zealand J. Bot. 17: 332 (1979); Notodanthonia monticola (Vickery) Veldkamp, Taxon 29: 296 (1980).

Type: New South Wales: Lithgow, 2 Jan 1936, Vickery s.n.; holo: NSW 1359; iso: K.

Austrodanthonia occidentalis (Vickery) H.P. Linder, comb. nov.

Danthonia occidentalis Vickery, Contrib. New South Wales Natl Herb. 1: 300 (1950). Synonyms: Rytidosperma occidentale (Vickery) Connor \& Edgar, New Zealand J. Bot. 17: 332 (1979); Notodanthonia occidentalis (Vickery) Veldkamp, Taxon 29: 297 (1980).

Type: Western Australia: Karrakatta, Nov. 1900, ex herb. W.V. Fitzgerald; holo: NSW 4389; iso: $\mathrm{K}$.

Austrodanthonia oreophila (H.P. Linder \& Walsh) H.P. Linder, comb. nov.

Rytidosperma oreophilum H.P. Linder \& Walsh, Muelleria 8: 283 (1995).

Synonym: Notodanthonia oreophila (H.P. Linder \& Walsh) H.P. Linder, Telopea 6: 616 (1996). Type: Australian Capital Territory: slopes of Mt Gingera, Bimberi Range, Pullen 3041; holo: CANB; iso: A, BO, K, L, MEL, NE, NSW.

Austrodanthonia penicillata (Labill.) H.P. Linder, comb. nov.

Arundo penicillata Labill., Pl. Nov. Holl. 1: 26 t.34 (1805).

Synonyms: Danthonia penicillata (Labill.) Beauv., Agrost.: 92, 153, 160 (1812); Danthonia racemosa R. Br. var. penicillata (Labill.) Benth., Fl. Austral. 7: 594 (1878); Notodanthonia penicillata (Labill.) Zotov, New Zealand J. Bot. 1: 122 (1963); Rytidosperma penicillatum (Labill.) Connor \& Edgar, New Zealand J. Bot. 17: 327 (1979).

Type: Tasmania: Labillardière; holo: FI-W, photo K.

Austrodanthonia pilosa (R. Br.) H.P. Linder, comb. nov. Basionym: Danthonia pilosa R. Br., Prodr. 177 (1810). 
Synonyms: Danthonia penicillata Labill. var. pilosa (R. Br.) Maiden \& Betche, Census N.S.W. Pl.: 22 (1916); Notodanthonia pilosa (R. Br.) Zotov, New Zealand J. Bot. 1: 118 (1963); Rytidosperma pilosum (R. Br.) Connor \& Edgar, New Zealand J. Bot. 17: 326 (1979). Type: New South Wales: Port Jackson, Brown, Bennett no. 6234; holo: BM; iso: K, MEL.

Austrodanthonia popinensis (D.I. Morris) H.P. Linder, comb. nov. Basionym: Danthonia popinensis D.I. Morris, Muelleria 7: 157 (1990). Synonym: Notodanthonia popinensis (D.I. Morris) H.P. Linder, Telopea 6: 616 (1996). Type: Tasmania: 0.5 km N of Kempton, Morris 8556; holo: HO 92651, iso: AD, NSW.

Austrodanthonia racemosa (R. Br.) H.P. Linder, comb. nov.

Basionym: Danthonia racemosa R. Br., Prodr.: 177 (1810).

Synonyms: Danthonia penicillata Labill. var. racemosa (R. Br.) Maiden \& Betche, Census N.S.W. Pl.: 22 (1916); Notodanthonia racemosa (R. Br.) Zotov, New Zealand J. Bot. 1: 121 (1963); Rytidosperma racemosum (R. Br.) Connor \& Edgar, New Zealand J. Bot. 17: 327 (1979). Type: New South Wales: Port Jackson, Brown, Bennett no. 6235; holo: BM; iso: K, fragm. MEL, fragm. PERTH.

Austrodanthonia racemosa (R. Br.) H.P. Linder var. obtusata (Benth.) H.P. Linder, comb. nov.

Basionym: Danthonia racemosa R. Br. var. obtusata Benth., Fl. Austral. 7: 594 (1878).

Synonyms: Rytidosperma racemosum var. obtusatum (Benth.) Connor \& Edgar, New Zealand J. Bot. 17: 332 (1979); Notodanthonia racemosa (R. Br.) Zotov var. obtusata (Benth.) Veldkamp, Taxon 29: 297 (1980).

Type: New South Wales: New England, Stuart s.n.; holo: K; iso: MEL.

Austrodanthonia remota (D.I.Morris) H.P. Linder, comb. nov. Basionym: Danthonia remota D.I. Morris, Muelleria 7: 160 (1990).

Synonym: Notodanthonia remota (D. Morris) H.P. Linder, Telopea 6: 617 (1996).

Type: Tasmania: summit of Hibbs Pyramid, 4 Jan 1984, Buchanan 2878; holo: HO 91392.

Austrodanthonia richardsonii (Cashmore) H.P. Linder, comb. nov.

Basionym: Danthonia richardsonii Cashmore, Comm. Aust., C.S.I.R., Bull. 69 Appendix 2: 72 (1932).

Synonyms: Rytidosperma richardsonii (Cashmore) Connor \& Edgar, New Zealand J. Bot. 17: 332 (1979); Notodanthonia richardsonii (Cashmore) Veldkamp, Taxon 29: 297 (1980). Type: South Australia: Werribee, 2 Dec 1932, Cashmore Ga63; holo: ADW (now at AD); iso: BM, K, NSW 4726, 1204).

Austrodanthonia setacea (R. Br.) H.P. Linder, comb. nov.

Basionym: Danthonia setacea R. Br., Prodr.: 177 (1810).

Synonyms: Rytidosperma setaceum (R. Br.) Connor \& Edgar, New Zealand J. Bot. 17: 332 (1979); Notodanthonia setacea (R. Br.) Veldkamp, Taxon 29: 297 (1980).

Type: Western Australia: King George's Sound, Brown, Bennett no. 6236; holo: BM; iso: BRI, K, fragm. MEL.

Austrodanthonia tenuior (Steud.) H.P. Linder, comb. nov.

Basionym: Plinthanthesis tenuior Steud., Syn. Pl. Gram.: 14 (1854).

Synonyms: Notodanthonia tenuior (Steud.) S.T. Blake, Contr. Queensland. Herb. 14: 3 (1972); Danthonia tenuior (Steud.) Conert, Senckenberg. Biol. 56: 163 (1975); Rytidosperma tenuius (Steud.) O.E. Erikss., A. Hansen \& Sunding, Fl. Macaronesia Checklist Vasc. Pl., pt. 1 edn. 2: 93 (1979).

Type: New South Wales: Port Jackson, D'Urville; holo: P. 


\section{Acknowledgments}

I would like to thank Tony Verboom for critically reading this paper, and correcting my Latin. The study was financed by the Foundation of Research Development, Pretoria.

\section{Reference}

Linder, H.P. and Verboom, G.A. (1996) Generic limits in the Rytidosperma (Danthonieae, Poaceae) complex. Telopea 6: 597-627.

Manuscript received 4 June 1997

Manuscript accepted 17 September 1997 